\title{
Incremental Expression of 14-3-3 Protein Beta/Alpha in Urine Correlates with Advanced Stage and Poor Survival in Patients with Clear Cell Renal Cell Carcinoma
}

\author{
Shiho Kaneko ${ }^{1 *}$, Kazumasa Matsumoto ${ }^{1}$,Satoru Minamida ${ }^{1}$, Takahiro Hirayama ${ }^{1}$, \\ Tetsuo Fujita ${ }^{1}$, Yoshio Kodera ${ }^{2}$, Masatsugu Iwamura ${ }^{1}$
}

\begin{abstract}
We investigated the urinary levels of 14-3-3 protein beta/alpha to evaluate their diagnostic significance with regard to clear cell renal cell carcinoma (ccRCC) and angiomyolipoma (AML). Urine samples from 91 patients with ccRCC, 16 patients with AML and 24 healthy volunteers were assessed. We used an enzyme-linked immunosorbent assay (ELISA) to quantify 14-3-3 protein beta/alpha levels in urine. Values were higher in patients with ccRCC than in those with AML and in healthy volunteers. High levels were associated with pathologic stage, lymph node status, distant metastasis and poor survival. Urinary levels of 14-3-3 protein beta/alpha were significantly increased in patients with small-sized carcinoma, irrespective of being less than $4.0 \mathrm{~cm}$ and $2.0 \mathrm{~cm}$, compared with levels in patients with AML. This study is the first to report that increased expression of 14-33 protein beta/alpha in urine is associated with advanced stage and poor survival in patients with ccRCC. In addition, urinary 14-3-3 protein beta/alpha may differentiate AML from RCC, even when small sized. These results suggest that examination of urinary 14-3-3 protein beta/alpha could serve as a diagnostic and prognostic marker in patients with ccRCC.
\end{abstract}

Keywords: 14-3-3 protein beta/alpha - renal cell carcinoma - diagnosis - ELISA

Asian Pac J Cancer Prev, 17 (3), 1399-1404

\section{Introduction}

Renal cell carcinoma ( $\mathrm{RCC}$ ) is the most common type of kidney cancer in adults, accounting for $3 \%$ of adult malignancies (Siegel et al., 2012). The incidence and mortality of RCC in Japanese have been increasing, partly because of the westernization of the lifestyle (Masakazu Washio et al., 2014). Approximately $80 \%$ of RCC cases are due to clear cell renal cell carcinoma (ccRCC) originating from the renal proximal tubule (Lipworth et al., 2006; Matsuura et al., 2011). Although patients with RCC are mainly treated with surgical excision because this type of cancer is resistant to traditional radiation and chemotherapy, approximately $30 \%$ of patients with RCC demonstrate locally advanced or metastatic lesions at the time of diagnosis (Weiss and Lin et al., 2006; Patil et al., 2010). One reason for delayed discovery in these cases was that RCC symptoms were absent and the cancer was detected incidentally during imaging studies such as computed tomography (CT) and ultrasonography. To address these clinical situations, an effective diagnostic tool is urgently required.

Certain proteins shed or secreted from cancerous tissues can reveal various phases of cancer development. Because the expression and amount of these proteins differ significantly from those that normally occur, the proteins represent targets of investigation for tumor markers in several bodily fluids. Recently, our study group identified 14-3-3 protein beta/alpha in cyst fluid at an early stage of cyst-associated RCC, using a proteomics approach (Satoru et al., 2011). The 14-3-3 proteins are down-regulated in human malignancies, leading to suppression of apoptosis (Aitken 1996; Fu et al., 2000; Zhao et al., 2011). Overexpression of 14-3-3 protein beta/alpha has been reported to stimulate cell proliferation and tumorigenesis (Takihara et al., 2000). In our previous study (Satoru et al., 2011), Western blotting was used to examine the 14-3-3 protein beta/alpha in cyst fluid, urine, serum and tissue in patients with RCC. The expression levels of 14-3-3 protein beta/alpha were higher in urine samples from patients with RCC than in those from healthy volunteers. The association of 14-3-3 protein beta/alpha expression with clinicopathologic findings in patients with RCC remains unknown.

To develop a diagnostic tool, we established an enzyme-linked immunosorbent assay (ELISA) to quantify 
14-3-3 protein beta/alpha levels in urine samples. In the present study, we investigated the urinary levels of 14-3-3 protein beta/alpha in patients with ccRCC, angiomyolipoma (AML) and healthy volunteers. We then compared the levels with clinicopathologic variables.

\section{Materials and Methods}

\section{Patients and samples}

Urine samples from 91 untreated patients with ccRCC of various stages and grades, 16 untreated patients with AML and 24 healthy volunteers were obtained between 2008 and 2013 at Kitasato University Hospital. Laboratory studies, chest X-ray, and pelvic computed tomography or magnetic resonance imaging were routinely done. All ccRCC patients underwent nephrectomy, and urine samples were obtained before and at least 1 (month) after the nephrectomy. Healthy volunteers did not have kidney cancer, other malignancies or urinary tract infection. Urine samples that were positive for leukocytes were excluded because 14-3-3 protein beta/alpha is overexpressed in cystitis (Satoru et al., 2011). Histological cancer types other than clear cell were also excluded. None of the patients had received chemotherapy or radiation before surgery. Immediately after sample collection, urine samples were centrifuged at $1,000 \times \mathrm{g}$ for $10 \mathrm{~min}$ at $4^{\circ} \mathrm{C}$ to remove cellular components and stored at $-80^{\circ} \mathrm{C}$ until use.

Since urinary concentration varied depending on an individual's physiological conditions such as hydration, we corrected urinary specific gravity by using a pocket refractometer (ATAGO, Japan). The refractometer was adjusted to 1.000 with high performance liquid chromatography (HPLC) grade water before use. A urine sample $(\sim 0.3 \mathrm{ml})$ was placed on the quartz window and the specific gravity was calculated, and all urine samples were then diluted with HPLC grade water to 1.004 , which was the lowest specific gravity in our samples.

ccRCC tissue samples were obtained from nephrectomy specimens. All specimens were histologically confirmed by pathologists after nephrectomy. The tumor histologic stage was defined by the recent classification criteria, which includes stage of disease and degree of tumor invasion (Greene et al., 2002). Normal tissue samples were obtained from the areas of benign lesions in patients with RCC.

The study protocol was approved by the Ethics Committee of the Kitasato University School of Medicine and Hospital, Kanagawa, Japan. All study participants provided written informed consent.

\section{ELISA for 14-3-3 protein beta/ alpha in urine}

The 14-3-3 protein beta/alpha in urine was measured by ELISA. The primary antibody (rabbit anti-human 14-3-3 $\beta$ polyclonal antibody, Sigma, St. Louis, MO, USA) was optimized at 1:10,000 in Can Get Signal 1 (TOYOBO, Osaka, Japan) and the secondary antibody (HRP goat anti-rabbit IgG, R\&D, USA) was optimized at 1:5,000 in Can Get Signal 2 (TOYOBO). The wells of a 96-well ELISA plate (Immulon, 4 HBX, Thermo Fisher Scientific, Rockford, IL, USA) were coated with urine samples diluted with coating buffer $(100 \mu \mathrm{l} /$ well; $50 \mathrm{mM}$ carbonate buffer, $\mathrm{pH}$ 9.6) and serial dilutions of standards (Recombinant Human YWHAB, Sino Biological Inc), then incubated at $37^{\circ} \mathrm{C}$ for 2 hours. After the solutions were removed, the blocking solution, $1 \%$ bovine serum albumin in tris-buffered saline (TBS), was placed in each well $(300 \mu \mathrm{l})$ and incubated at $4^{\circ} \mathrm{C}$ overnight. After 5 washes with TBST (TBS with $0.05 \%$ Tween 20 ), primary antibody was added at $100 \mu \mathrm{l} /$ well and incubated at room temperature (RT) for 45 minutes. After 5 washes of the wells, the secondary antibody was added (100 $\mu \mathrm{l} /$ well) and incubated at RT for 45 minutes. Following another 5 washes with TBST, $100 \mu$ of tetramethylbenzidine (TMB) substrate buffer was added to each well and incubated at RT for 8 minutes. Finally, $100 \mu$ of 0.18 $\mathrm{M} \mathrm{H}_{2} \mathrm{SO}_{4}$ was added to each well to stop the enzyme reaction. Absorbance was read at $450 \mathrm{~nm}$ and $540 \mathrm{~nm}$ of wavelength on a microplate reader (ELX800 enzyme linked immunosorbent detector; Dio-Tek Instruments Inc., Winooski, VT, USA). The standard curve was determined using the absorbance of the standard solution.

\section{Immunohistochemical staining}

Tissues were fixed in $10 \%$ neutral buffered formalin, dehydrated with a series of graded concentrations of ethanol and embedded in paraffin, and tissue sections were stained with hematoxylin and eosin for histologic examination. A paraffin block used for routine pathologic diagnosis was selected for each case, and 3- $\mu$ m-thick paraffin sections were prepared for immunohistochemical analysis.

To explore where the 14-3-3 protein beta/alpha was expressed, we performed immunohistochemistry staining on representative ccRCC tissues, normal renal tissues, and AML tissues. For 14-3-3 protein beta/ alpha immunohistochemistry, sections on glass slides were deparaffinized and washed with water. Epitopes were demasked using heat-induced epitope retrieval in an autoclave ( $\mathrm{pH} 6$ citrate buffer solution, 20 minutes at $105^{\circ} \mathrm{C}$ ). Sections were then incubated in $3 \% \mathrm{H}_{2} \mathrm{O}_{2}$ methanol for 15 minutes at RT to remove the endogenous peroxidase. After being washed in water, sections were blocked for 10 minutes with Protein Block Serum-Free (Dako, Glostrup, Denmark) and then incubated for 60 minutes at RT with 14-3-3 $\beta$ polyclonal antibody $(1: 1,000$, rabbit anti-human 14-3-3 $\beta$ polyclonal antibody, Sigma). The sections were subsequently rinsed in phosphatebuffered saline (PBS), incubated for 30 minutes at RT with Dako ChemMate ENVISION kit/HRP(Dako). After being washed in PBS, 14-3-3 protein beta/alpha-positive cells were visualized by incubating sections with Liquid diaminobenzidine (DAB) and Substrate Chromogen System (Dako). The sections were counterstaining with hematoxylin.

\section{Statistical analysis}

Sex, tumor pathologic stage $(<\mathrm{pT} 3 v s \geq \mathrm{pT} 3)$, grade (grade $1 v s$ grade $2 v s$ grade 3 ), lymph node status (N0 vs N1) and distant metastasis (M0 vs M1) were evaluated as dichotomized variables. The area under the curve (AUC) and best cutoff point were calculated by employing receiver operating characteristic (ROC) analysis. The 
Urinary 14-3-3 Protein Beta/Alpha Correlates with Advanced Stage and Poor Survival in Patients with Clear Cell RCC

Kaplan Meier method was used to calculate survival functions, and differences were assessed with the log rank statistic. Statistical significance in this study was set at $\mathrm{p}<0.05$. All reported $\mathrm{p}$ values are two-sided. All statistics were analyzed using GraphPad Prism (version 6).

\section{Results}

Expression levels of 14-3-3 protein beta/alpha in urine

The mean and median ages of ccRCC patients were 63 and 64 years, respectively (range: $34-88$ years). The clinicopathologic characteristics in patients with ccRCC are shown in Table 1. Urinary 14-3-3 protein beta/alpha levels were significantly higher in patients with ccRCC than in healthy volunteers and AML patients $(282.4$, 125.2 and $103.6 \mathrm{ng} / \mathrm{ml}$, respectively, both $\mathrm{p}<0.0001$, Figure 1). High levels of 14-3-3 protein beta/alpha expression were associated with pathologic stage, lymph node status and distant metastasis $(\mathrm{p}=0.0025,0.042$ and 0.046 , respectively, Table 1). Urinary levels of 14-3-3 protein beta/alpha were significantly increased in patients with small ccRCC, irrespective of being less than 4.0 $\mathrm{cm}$ and $2.0 \mathrm{~cm}$, compared to those of healthy volunteers (274.8 and $261.9 \mathrm{ng} / \mathrm{ml}, \mathrm{p}=0.0005,0.0031$, respectively, Figure 2) and to those of AML patients $(\mathrm{p}<0.0001$ and 0.0005 , respectively, Figure 2). However, no significant differences were observed between urinary levels of 143-3 protein beta/alpha and the other factors, including sex and pathologic grade. No significant differences existed between urinary levels of 14-3-3 protein beta/ alpha in healthy volunteers and AML patients $(\mathrm{p}=0.38$, Figure 1). Urinary levels of 14-3-3 protein beta/alpha levels significantly decreased after surgery compared to preoperative samples $(64.3 \mathrm{ng} / \mathrm{ml}, \mathrm{p}=0.003$, Figure 3$)$.

We examined the availability of 14-3-3 protein beta/

Table 1. Association of Preoperative Urinary 143-3 Protein Beta/Alpha Levels with Clinical and Pathological Characteristics

\begin{tabular}{|c|c|c|c|}
\hline & $\begin{array}{l}\text { No. of } \\
\text { patients } \\
(\%)\end{array}$ & $\begin{array}{l}\text { 14-3-3 protein beta/ } \\
\text { alpha levels } \\
(\mathrm{ng} / \mathrm{ml})\end{array}$ & p Value* \\
\hline \multicolumn{4}{|l|}{ Sex } \\
\hline Male & $67(74)$ & 287.6 & 0.97 \\
\hline Female & $24(26)$ & 278 & \\
\hline \multicolumn{4}{|c|}{ Pathological grade } \\
\hline 1 & $36(39)$ & 283.1 & $0.19 * *$ \\
\hline 2 & $49(54)$ & 265.7 & \\
\hline 3 & $6(7)$ & 414.9 & \\
\hline \multicolumn{4}{|c|}{ Pathological stage } \\
\hline $\mathrm{pT} 1,2$ & $75(82)$ & 262.5 & 0.0025 \\
\hline pT3,4 & $16(18)$ & 391 & \\
\hline \multicolumn{4}{|c|}{ Regional lymph nodes*** } \\
\hline No & $83(95)$ & 273.2 & 0.042 \\
\hline N1 & $4(5)$ & 457.6 & \\
\hline \multicolumn{4}{|c|}{ Distant metastasis } \\
\hline M0 & $82(92)$ & 273.6 & 0.048 \\
\hline M1 & $9(8)$ & 386.1 & \\
\hline
\end{tabular}

*Mann-Whitney U test; **Kruskal-Wallis test; ***Four patients with unknown status of lymph node alpha as a biomarker for distinguishing ccRCC patients from healthy controls or AML patients. In ccRCC patients and healthy controls, at a cutoff point of $261.1 \mathrm{ng} / \mathrm{ml}$, the sensitivity and specificity of 14-3-3 protein beta/alpha were $46.2 \%$ (95\% CI: 35.6-56.9) and 95.8\% (95\% CI: 78.9-99.9), respectively (Figure 4A). The AUC-ROC levels were 0.751 (95\% CI: $0.66-0.85$, p<0.0005). In patients with ccRCC and AML, at a cutoff point of 196.1 $\mathrm{ng} / \mathrm{ml}$, the sensitivity and specificity of $14-3-3$ protein beta/alpha were $60.4 \%$ (95\% CI: 49.6-70.5) and $93.8 \%$ (95\% CI: 69.8-99.8), respectively (Figure 4B). The AUCROC levels were 0.815 (95\% CI: $0.72-0.91$, p<0.0001).

\section{Survival}

Of 91 patients, 4 patients (4\%) experienced disease recurrence and 7 were dead at the time of analysis. While 5 patients $(71 \%)$ died of metastatic ccRCC, $2(29 \%)$ died of other causes and had no evidence of disease at the time

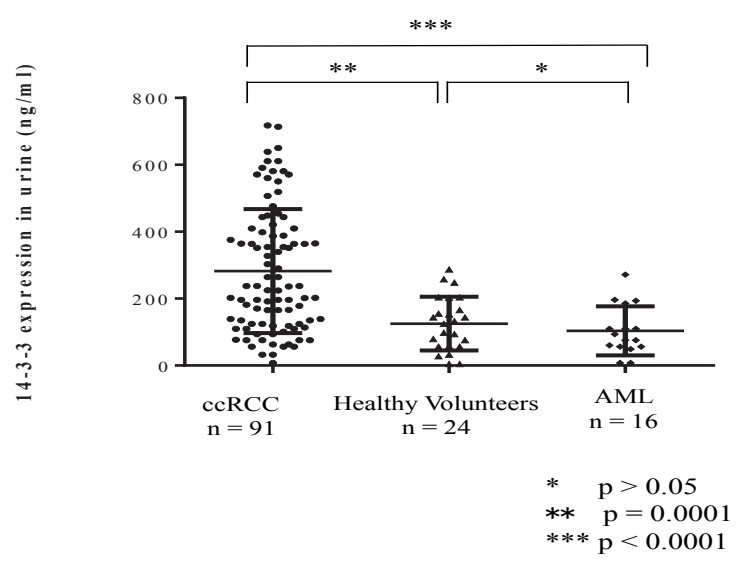

Figure 1. Urinary Levels of 14-3-3 Protein Beta/Alpha Expression in Patients with Clear Cell Renal Cell Carcinoma, Healthy Volunteers and Angiomyolipoma (AML). Horizontal lines indicate medians; $\mathrm{n}=$ number of subjects

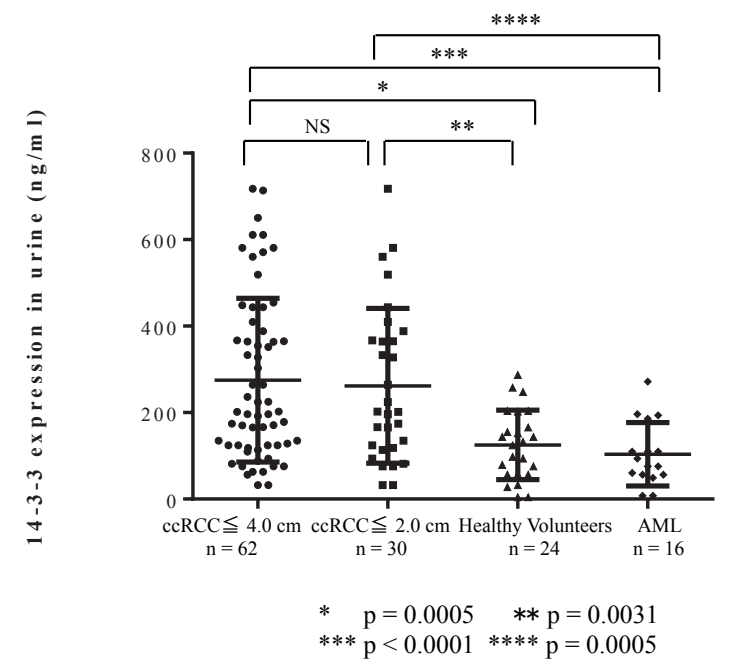

Figure 2. Urinary Levels of 14-3-3 Protein Beta/Alpha Expression in Patients with Small Renal Cancer, Healthy Volunteers and Angiomyolipoma (AML). Horizontal lines indicate medians; $n=$ number of subjects 


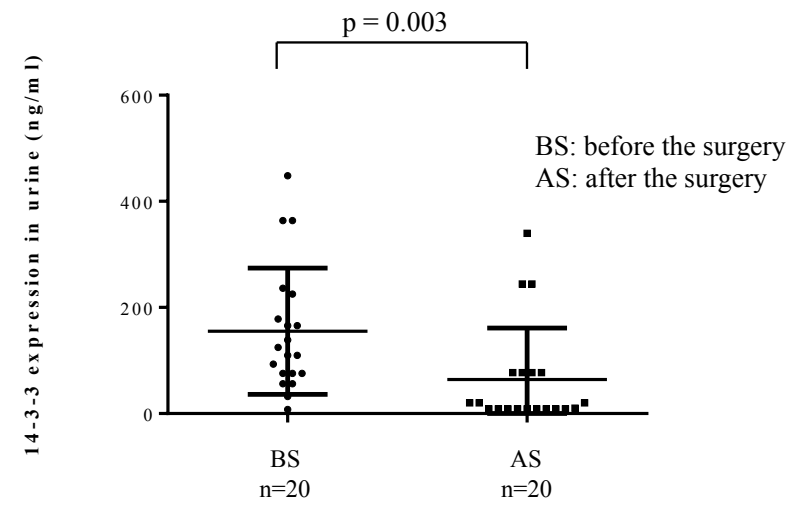

Figure 3. Pre- and Postoperative Urinary Levels of 14-3-3 Protein Beta/Alpha In Patients With Clear Cell Renal Cell Carcinoma. Horizontal lines indicate medians; $\mathrm{n}=$ number of subjects

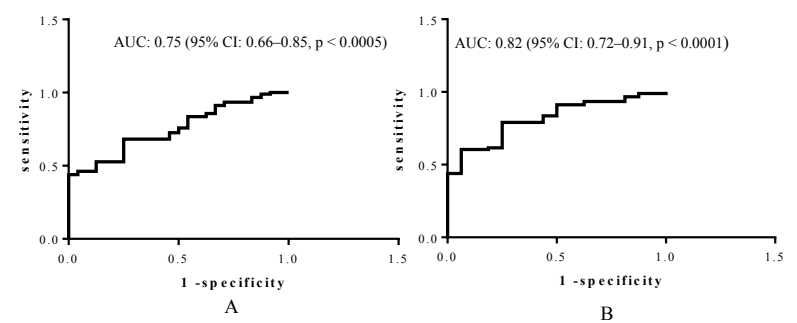

Figure 4. Receiver Operating Characteristic (ROC) Curves For Clear Cell Renal Cell Carcinoma Versus Healthy Volunteer (A) and Clear Cell Renal Cell Carcinoma Versus Angiomyolipoma (AML) (B). The area under the curve (AUC)-ROC levels were 0.75 and 0.82 ,
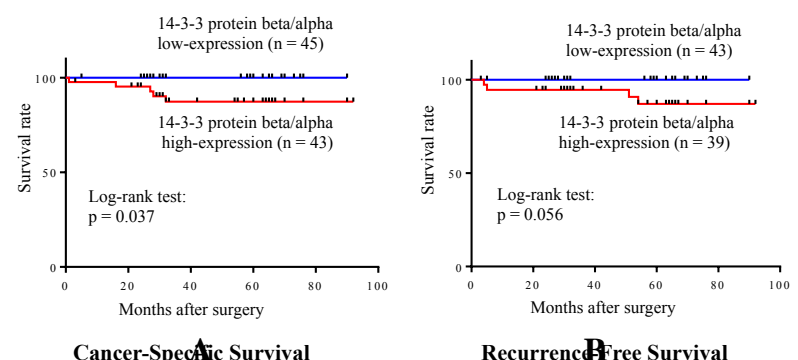

Figure 5. Probability of Cancer-specific Survival (A) and Recurrence-free Survival (B) after Nephrectomy based on the Urinary Levels of 14-3-3 Protein Beta/ Alpha

of death. The mean and median follow-up times were 47.8 and 56 months, respectively (range: 3-92 months) for the surviving patients at the last follow-up. Using Kaplan-Meier analysis, patients with urinary 14-3-3 protein beta/alpha levels above the median $(288.7 \mathrm{ng} / \mathrm{ml})$ had a significantly increased probability of cancer-specific survival ( $p=0.037$; Figure $5 \mathrm{~A}$ ), but not of recurrence-free survival $(\mathrm{p}=0.056$; Figure $5 \mathrm{~B})$.
A

B

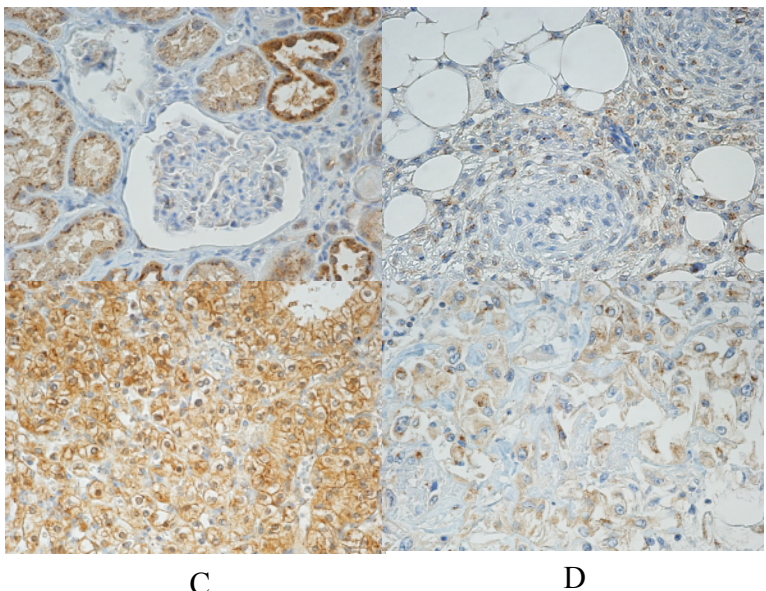

Figure 6. Immunohistochemical Staining of 143-3 Protein Beta/Alpha in Clear Cell Renal Cell Carcinoma (ccRCC) Tissue, Normal Renal Cortex and Angiomyolipoma (AML) Tissue (×400). (A) Normal site of ccRCC tissue, (B) tumor region of AML, (C) pT1 ccRCC with high expression of 14-3-3 protein beta/alpha, (D) pT3 ccRCC with low expression of 14-3-3 protein beta/alpha

Immunohistochemical staining for 14-3-3 proteins in RCC

Representative staining patterns in ccRCC, AML and normal renal tissues are shown in figure 6. 14-3-3 Protein beta/alpha is expressed in distal renal tubules but not in glomeruli in normal tissue (Figure 6A). Expression in AML tissues was low (Figure 6B). In cancerous tissue, 14-3-3 protein beta/alpha was expressed in tumor cells but not in interstitial cells (Figure 6C and D).

\section{Discussion}

Patients with metastatic RCC have a poor prognosis, with a 5-year survival rate of 5\% compared with $50 \%$ to $95 \%$ in patients with localized disease. Because of the cancer's resistance to radiation and chemotherapy, therapeutic options for metastatic or recurrent RCC often can be offered only molecular target therapy (Patil et al., 2010). In contrast, minimally invasive therapies such as laparoscopic nephrectomy or partial nephrectomy are options for patients with early stage of RCC (Amato 1996). These therapies have many benefits, including small operative scar, minimal pain and short hospitalization. However, small lesions are incidentally detected in the context of unrelated examinations. Early detection of RCC is important not only for better therapeutic options but also for a better prognosis.

Although many studies have reported biomarkers for RCC in serum and urine (Wei C et al., 2013; Yu ZH et al., 2013; Wen RM et al., 2015), none of them have been clinical applied (Ngo et al., 2014). One of the candidates is 14-3-3 protein beta/alpha in urine. Our previous study showed that 14-3-3 protein beta/alpha was overexpressed in cyst fluid from cyst-associated RCC (Satoru et al., 2011). The 14-3-3 proteins have a molecular weight of approximately $30 \mathrm{kDa}$ and mainly occur in the synapses 
Urinary 14-3-3 Protein Beta/Alpha Correlates with Advanced Stage and Poor Survival in Patients with Clear Cell RCC

and neuronal cytoplasm in the human brain (Mhawech 2005). However, they are present in almost all tissues (Aitken 1996) and play an important role in various processes, including cell proliferation, cell apoptosis and angiogenesis (Takihara et al., 2000; Sugiyama et al., 2003; Liou et al., 2008; Morrison 2009). In this study, we confirmed urinary levels of 14-3-3 protein beta/alpha were higher in patients with ccRCC than in healthy volunteers by using conventional ELISA.

Recently, the overexpression of 14-3-3 protein beta/ alpha was reported to be associated with a variety of malignant diseases (Qi et al., 2005; Musholt et al., 2006; Yang et al., 2009; Liu et al., 2011; Wu et al., 2012). Qi et al (Qi et al., 2005) indicated that 14-3-3 protein beta/alpha was frequently expressed in lung cancer but not in normal tissue, showing that it might be a useful marker for lung cancer diagnosis and a target for therapy. Liu et al (Liu et al., 2011) demonstrated that the expression levels of 14-3-3 protein beta/alpha were positively correlated not only with cancer progression but also with extrahepatic metastasis and worse survival in hepatocellular carcinoma (HCC) cases. They suggested that 14-3-3 protein beta/ alpha might become a novel prognostic biomarker to identify HCC patients at high risk of metastasis. These reports used immunohistochemical staining to provide evidence that high expression of 14-3-3 protein beta/ alpha plays an important role in malignant tumor growth and progression. We found that high expression of urinary 14-3-3 protein beta/alpha levels was associated with pathologic stage and metastasis in lymph nodes or distant organs and with poor survival. In addition, 14-33 protein beta/alpha expression was shown in surgical ccRCC specimens. These findings could be explained by the functional role of 14-3-3 protein beta/alpha, namely, resistance to apoptosis. At an advanced stage of ccRCC, more 14-3-3 protein beta/alpha may be produced, leading to poor survival.

Importantly, we also found that urinary 14-3-3 protein beta/alpha expression could differentiate ccRCC from AML, the benign tumor of kidney. AML is usually asymptomatic and often does not require intervention (Mues et al., 2010). Indications for intervention include suspicion of malignancy, risk of rupture, spontaneous hemorrhage and pain (Bissler and Kingswood, 2004). Although most cases of AML are diagnosed by identifying the presence of macroscopic fat component using computed tomography or magnetic resonance imaging, it can be difficult to differentiate AML from ccRCC (Jinzaki et al., 1997). Since urinary levels of 14-3-3 protein beta/ alpha in patients with ccRCC were significantly higher than those of AML patients, the protein may be a useful diagnostic marker to distinguish renal tumors.

Small renal masses (SRMs) are generally defined as solid enhancing tumors up to $4.0 \mathrm{~cm}$ in maximal diameter within the kidney, and they account for up to $66 \%$ of all renal tumors (Nguyen et al., 2006) that are incidentally diagnosed in clinical practice. There are currently no clinical or radiographic features that accurately predict histological findings, and at least $20 \%$ of radiologically suspicious SRMs are in fact benign masses at final pathological examination (Bissler and Kingswood, 2004;
Frank et al., 2003). We found that urinary 14-3-3 protein beta/alpha levels were higher in patients with ccRCC, even at a small size, than in healthy control. In addition, urinary 14-3-3 protein beta/alpha levels in patients with small-sized ccRCC were also significantly higher than in those with AML, indicating that urinary 14-3-3 protein beta/alpha could potentially help identify SRMs that are malignant. In patients at high-risk of developing RCC, including heavy smokers (Washio M et al., 2013), individuals undergoing hemodialysis (Lowrance WT et al., 2014), and individuals with a positive family history, Von Hippel-Lindau disease or immunosuppression, screening urine samples for 14-3-3 protein beta/alpha may be beneficial. Furthermore, active surveillance might be a valid alternative for selected cases such as elderly patients or those with comorbidities when the urinary 14-3-3 protein beta/alpha levels would otherwise be low.

The present study has some limitations. First, our study contained only 91 patients with ccRCC ( $73 \%$ of them were pT1) and 16 patients with AML. Second, 14-3-3 protein beta/alpha was not measured in other urological benign and malignant lesions or in other malignancies. However, on the basis of our analyses, 14-3-3 protein beta/alpha has the potential not only to be a diagnostic biomarker but also a prognostic marker, and it could prevent unnecessary surgeries in patients with SRMs in the future. This study showed that increased urinary 14-3-3 protein beta/alpha levels are significantly associated with ccRCC. Finally, although postoperative urinary 14-3-3 protein beta/alpha levels were significantly decreased in 20 patients, we need to investigate more postoperative urine samples in patients treated with nephrectomy. We established an ELISA for 14-3-3 protein beta/alpha, which was not commercially available, to quantify the protein levels in urine. Urine is an ideal sample for a biomarker because it can be obtained noninvasively and ELISA enabled simultaneous quantification of multiple urine samples. The study limitations need to be overcome prior to using 14-3-3 protein beta/alpha as a biomarker of ccRCC in a clinical setting.

In conclusions, We found that 14-3-3 protein beta/ alpha was higher in patients with ccRCC than in healthy volunteers. In addition, the expression levels of 14-3-3 protein beta/alpha in urine were associated with advanced stage and poor prognosis in patients with ccRCC. Furthermore, urinary 14-3-3 protein beta/alpha may differentiate even small-sized RCC from AML. These findings suggested that 14-3-3 protein beta/alpha could have a potential role in detection and as a prognostic factor for ccRCC. Further studies are warranted to verify the usefulness of urinary levels of 14-3-3 protein beta/alpha in a real-world clinical scenario.

\section{Acknowledgements}

This study was supported in part by a Grant-in-Aid for Scientific Research C (15K10607) from The Japan Society for the Promotion of Science (to K. Matsumoto). We thank Yuriko Shimizu, MS, for technical support of enzymelinked immunosorbent assay at Kitasato University. 


\section{References}

Aitken A (1996). 14-3-3 and its possible role in co-ordinating multiple signaling pathways. Trends Cell Biol, 6, 341-7.

Amato RJ (2005). Renal cell carcinoma: review of novel single agent therapeutics and combination regimens. Ann Oncol, 16, 7-15.

Bissler JJ, Kingswood JC (2004). Renal angiomyolipomata. Kidney Int, 66, 924-34.

Frank I, Blute ML, Cheville JC, et al (2003). Solid renal tumors: an analysis of pathological features related to tumor size. $J$ Urol, 170, 2217-20.

Fu H, Subramanian RR, Masters SC (2000). 14-3-3 proteins: structure, function, and regulation. Annu Rev Pharmacol Toxicol, 40, 617-47.

Greene FL, Page DL, Fleming ID, et al (2002). AJCC Cancer Staging Manual, $6^{\text {th }}$ edn. Springer, New York.

Jinzaki M, Tanimoto A, Narimatsu Y, et al (1997). Angiomyolipoma: imaging findings in lesions with minimal fat. Radiol, 205, 497-502.

Liou JY, Wu CC, Chen BR, et al (2008). Nonsteroidal antiinflammatory drugs induced endothelial apoptosis by perturbing peroxisome proliferator-activated receptor-delta transcriptional pathway. Mol Pharmacol, 74, 1399-1406.

Lipworth L, Tarone RE, McLaughlin JK (2006). The epidemiology of renal cell carcinoma. J Urol, 176, 2353-8.

Liu TA, Jan YJ, Ko BS, et al (2011). Increased expression of 14$3-3 \beta$ promotes tumor progression and predicts extrahepatic metastasis and worse survival in hepatocellular carcinoma. Am J Pathol, 179, 2698-708.

Lowrance WT, Ordonez J, Udaltsova N, et al (2014). CKD and the risk of incident cancer. J Am Soc Nephrol, 25, 2327-34

Masakazu W, Mitsuru M, Kazuya M, et al (2014). Risk Factors for Renal Cell Carcinoma in a Japanese Population. Asian Pac J Cancer Prev, 15, 9065-70.

Matsuura K, Nakada C, Mashio M, et al (2011). Downregulation of SAV1 plays a role in pathogenesis of high-grade clear cell renal cell carcinoma. BMC Cancer, 11, 523-32.

Mhawech P (2005). 14-3-3 proteins-an update. Cell Res, 15, 228-236.

Morrison DK (2009). The 14-3-3 proteins: integrators of diverse signaling cues that impact cell fate and cancer development. Trends Cell Biol, 19, 16-23.

Mues AC, Palacios JM, Haramis G, et al (2010). Contemporary experience in the management of angiomyolipoma. $J$ Endourol, 24, 1883-6.

Musholt TJ, Brehm C, Hanack J, et al (2006). Identification of differentially expressed genes in papillary thyroid carcinomas with and without rearrangements of the tyrosine kinase receptors RET and/or NTRK1. J Surg Res, 131, 15-25.

Ngo TC, Wood CG, Karam JA (2014). Biomarkers of renal cell carcinoma. Urol Oncol, 32, 243-51.

Nguyen MM, Gill IS, Ellison LM (2006). The evolving presentation of renal carcinoma in the United States: trends from the Surveillance, Epidemiology, and End Results Program. J Urol, 176, 2397-400.

Patil S, Ishill N, Deluca J, et al (2010). Stage migration and increasing proportion of favorable-prognosis metastatic renal cell carcinoma patients. Cancer, 116, 347-54.

Qi W, Liu X, Qiao D, et al (2005). Isoform-specific expression of 14-3-3 proteins in human lung cancer tissues. Int J Cancer, 113, 359-63.

Satoru M, Masatsugu I, Yoshio K, et al (2011). 14-3-3 Protein beta/alpha as a urinary biomarker for renal cell carcinoma: proteomic analysis of cyst fluid. Anal Bioanal Chem, 401, 245-52.

Siegel R, Naishadham D, Jemal A (2012). Cancer statistics. CA
Cancer J Clin, 62, 10-29.

Sugiyama A, Miyagi Y, Komiya Y, et al (2003). Forced expression of antisense14-3-3beta RNA suppresses tumor cell growth in vitro and in vivo. Carcinogenesis, 24, 1549-59.

Takihara Y, Matsuda Y, Hara J (2000). Role of the beta isoform of 14-3-3 proteins in cellular proliferation and oncogenic transformation. Carcinogenesis, 21, 2073-7.

Washio M, Mori M, Mikami K, et al (2013). Cigarette smoking and other risk factors for kidney cancer death in a Japanese population: Japan collaborative cohort Study for Evaluation of Cancer Risk (JACC study). Asian Pac J Cancer Prev, 14, 6523-8.

Wei C, Lai YQ, Li XX,Ye JX (2013). TGF-beta-activated kinase-1: a potential prognostic marker for clear cell renal cell carcinoma. Asian Pac J Cancer Prev, 14, 315-20.

Weiss RH, Lin PY (2006). Kidney cancer: identification of novel targets for therapy. Kidney Int, 69, 224-32.

Wen RM, Zhang YJ, Ma S, et al (2015). Preoperative neutrophil to lymphocyte ratio as a prognostic factor in patients with non-metastatic renal cell carcinoma. Asian Pac J Cancer Prev, 16, 3703-8.

Wu Q, Liu CZ, Tao LY, et al (2012). The clinicopathological and prognostic impact of 14-3-3 protein isoforms expression in human cholangiocarcinoma by immunohistochemistry. Asian Pac J Cancer Prev, 13, 1253-9.

Yang X, Cao W, Lin H, et al (2009). Isoform-specific expression of 14-3-3 proteins in human astrocytoma. J Neurol Sci, 276, 54-9.

Yu ZH, Zhang Q, Wang YD, et al (2013). Overexpression of cyclooxygenase-1 correlates with poor prognosis in renal cell carcinoma. Asian Pac J Cancer Prev, 14, 3729-4.

Zhao J, Meyerkord CL, Du Y, et al (2011). 14-3-3 proteins as potential therapeutic targets. Semin Cell Dev Biol, 22, 705-12. 